\title{
Percepções de especialistas sobre o processo de mudança na contabilidade de custos no setor público do Brasil
}

\author{
Renato Pereira Monteiro \\ Doutorando em Contabilidade pelas Universidades de Aveiro e Universidade do Minho \\ Portugal \\ Portugal \\ E-mail: renatomonteir@gmail.com \\ Joaquim Carlos da Costa Pinho \\ Professor na Universidade de Aveiro \\ Portugal \\ E-mail: cpinho@ua.pt
}

\section{RESUMO}

O estudo teve por objetivo responder a questionamentos como: (1) de que maneira os especialistas entendem o processo de implantação da NBC T 16.11?; (2) qual é a utilidade e importância desse sistema?; (3) quais são as principais dificuldades encontradas?; (4) qual é a perspectiva de futuro? Para este trabalho, utilizou-se um questionário direcionado aos especialistas em contabilidade do setor público do Brasil. Nove especialistas responderam, e a análise dos dados permitiu constatar que seu estágio de implantação ainda é muito embrionário. Mesmo após o regramento, são raros os entes públicos que implantaram ou tentaram a implantação, a qual é considerada o início de um longo processo. Existe concordância de que elaé importante no contexto atual da gestão pública. Seus contributos são entendidos como comparações de resultados, serviços, setores, entidades, melhoraria da gestão e a utilização dos recursos, efetividade, eficiência, eficácia, planejamento, tomada de decisão, controle social, função alocativa (orçamento) e transparência pública. As dificuldades apontadas foram: falta de sistemas informáticos adequados, baixa remuneração dos profissionais de contabilidade, falta de treinamento e inexistência de regras punitivas para a não adoção da referida Norma.

Palavras-Chave: Contabilidade de Custos. Setor Público. NBC T 16.11. NBCASP.

Specialists' perceptions on the cost accounting's change process in Brazil's public sector

\section{ABSTRACT}

This study sought to answer: 1) how specialists perceive the NBC T16.11's implementation process? 2) What is the usefulness and importance of this system? 3) What are the main difficulties found? 4) What are the future prospects? A questionnaire 
Percepções de especialistas sobre o processo de mudança na contabilidade de custos no setor público do Brasil

Renato Pereira Monteiro, Joaquim Carlos da Costa Pinho

directed at accounting specialists in Brazil's public sector was used. Nine specialists responded. The data analysis has shown that its implementation is still at a very early stage. Even after it was regulated, there had been a very low number of public entities that have implemented or that have tried to implement it. Implementation is considered to be the beginning of a long process. There is a general agreement that it is important in the current context of public management. Its contributions are perceived as being comparisons of results, services, sectors, entities, improving management and the use of resources, effectiveness, efficiency, efficacy, planning, decision making, social control, allocation function (budget) and public transparency. The difficulties highlighted were the lack of appropriate computer systems, low pay of accounting professionals, lack of training, inexistence of punitive measures for those who don't adopt it.

Keywords: Cost Accounting. Public Sector. NBC T 16.11. NBCASP.

\section{INTRODUÇÃO}

A contabilidade do setor público tem passado por modificações significativas desde 2000. O processo reformista da contabilidade pública foi motivo de diversas pesquisas até o presente momento. Esses estudos tratam de aspectos referentes ao processo de harmonização ou implantação da contabilidade por competência. O processo de harmonização e padronização foi objeto de pesquisas como as de Benito, Brusca e Montesinos (2007) e Dasí, Montesinos e Murgui (2013). Por outro lado, a implementação da contabilidade por competência também tem motivado muitas pesquisas que, por vezes, utilizam comparação entre países (Rossi, Cohen, Caperchione, \& Brusca, 2016) ou buscam retratar a realidade da União Europeia, como em Pina, Torres e Yetano (2009) e Oulasvirta (2014).

Nesse conjunto de alterações e modificações, também foi incorporada a implantação da contabilidade de gestão, um dos focos da New Public Magament NPM, como se apresenta em Van-Peursem e Eggleton (2015). As pesquisas anteriores derivam das vantagens da implantação que, conforme Arnaboldi e Lapsley (2009), estão centradas em fornecer informações como custo total, que poderá ser útil para melhorar a gestão interna. Entre as vantagens constatadas para o uso da informação de custos estão: orçamento e alocação de recursos financeiros, decisões de outsourcing, avaliação dos custos de serviços e prestação de contas interna. 
Percepções de especialistas sobre o processo de mudança na contabilidade de custos no setor público do Brasil

Renato Pereira Monteiro, Joaquim Carlos da Costa Pinho

No Brasil, também cresce o interesse pelas pesquisas no setor público, em especial pela edição das Normas Brasileiras de Contabilidade Aplicadas ao Setor Público - NBCASP - em 2008 e, posteriormente, a edição do Manual de Contabilidade Aplicado ao Setor Público - MCASP. As NBCASPs foram complementadas em 2011 pela NBC T 16.11, que trata do Subsistema de Custos Aplicado ao Setor Público. Essa norma sofreu, em 2013, um conjunto de alterações significativas, com revisão de alguns aspectos criticados e preenchimento de lacunas que existiam na opinião dos usuários e técnicos.

Esses novos dispositivos revelaram a importância dos aspectos patrimoniais e de gestão para a contabilidade pública, sem perder de vista as peculiaridades dos aspectos orçamentários. As recentes modificações complementadas pelas alterações na estrutura do plano de contas, na reestruturação dos Anexos da Lei no 4.320/64 e nas mudanças da Lei ํㅜ 6.404/76 do setor privado, pelas Leis o 11.638/07 e 11.941/09, estão evidenciando aproximação entre a contabilidade do setor público e a do setor privado, e ambas estão em busca da convergência com as normas internacionais.

Estudos anteriores, como o de Drehmer, Raupp e Tezza (2016) e Lovato, Oliveira, Guimarães e Catapan (2016), reconheceram as dificuldades do setor público em implantar o sistema de contabilidade de custos. Diante desse cenário, este estudo busca responder, principalmente: (1) Como os especialistas entendem o processo de implantação da NBC T 16.11?; (2) Qual é a utilidade e importância desse sistema?; (3) Quais são as principais dificuldades encontradas?; (4) Qual é a perspectiva de futuro?

$\mathrm{Na}$ próxima seção, apresenta-se a revisão da literatura sobre contabilidade de custos no Brasil, aspectos da NBC T 16.11 e barreiras para a implantação na visão de diferentes autores. A metodologia é apresentada na seção 3. Na seção 4, realiza-se análise e discussão dos resultados e, na seção 5, são apresentadas as conclusões do estudo. 
Percepções de especialistas sobre o processo de mudança na contabilidade de custos no setor público do Brasil Renato Pereira Monteiro, Joaquim Carlos da Costa Pinho

\section{REVISÃO DA LITERATURA}

Esta seção apresentará os principais conceitos relativos à contabilidade do setor público no Brasil, fazendo uma retomada de suas modificações nos últimos anos e caracterizando seus principais elementos e como eles repercutiram na realidade dessas organizações.

\subsection{Contabilidade de custos no Brasil}

Percebe-se avanço significativo da contabilidade pública no país. A contabilidade pública deve ser o meio para efetivo controle da sociedade, servindo de base para geração de informações que permitam o acompanhamento, pelo conjunto da sociedade, das contas públicas, da gestão do seu patrimônio e da aplicação dos recursos públicos. Com esse enfoque, cresceu a necessidade de se ter também uma contabilidade voltada para controle, gestão e informação de custos (Monteiro, Pereira, \& Souza, 2015) e (Padrones, Santos, \& Colares, 2017).

As recentes alterações na contabilidade pública brasileira, em sua origem, decorrem do movimento de convergência aos padrões internacionais e são formalizadas pelo Internacional Accounting Standards Board - IASB -, como forma de melhorar a informação. Neste sentido, Vicente, Morais e Platt (2012) afirmam que a convergência, inicialmente, estava vinculada ao setor privado e foi estendida ao setor público, visando à possibilidade de comparar resultados obtidos pelos entes governamentais e melhora da qualidade da informação e da evidenciação contábil.

Até o advento das novas normas, não existia um conjunto de normativos que regrassem especificamente os diversos aspectos da contabilidade pública nacional, gerando muitas vezes interpretações equivocadas e desiguais numa mesma unidade federativa (Monteiro, Pereira, \& Pereira, 2014). Apesar de a Lei $n^{\circ} 4.320 / 64$ contemplar aspectos como eventuais demonstrações que caberiam à contabilidade pública evidenciar, em outros quesitos foi incipiente, destacando-se a falta de um detalhamento 
de como deveria funcionar a apuração de custos. O primeiro avanço significativo foi sentido com a Lei de Responsabilidade Fiscal - LRF.

Com o advento da LRF e dos preceitos da NPM, a temática dos custos no setor público passou a ser amplamente discutida, principalmente pela reconhecida falta de cultura de gestão e controle dos custos por parte dessas instituições e de seus agentes (Mausse Souza, 2008). Já afirmava Alonso (1999) que, no setor privado, existem diversificadas experiências com sistemas de custos, todavia, no setor público, essa realidade não era igual.

$\mathrm{Na}$ opinião de Pangnani e Oliveira (2009), no setor público os custos merecem uma abordagem mais ampla em relação à contabilidade de custos praticada no setor privado, pois envolve critérios e exigências característicos da contabilidade pública, visto que a do setor privado tem como característica a preocupação com produtividade e eficácia nos resultados de produtos e serviços de mercado.

Segundo Slomski, Camargo, Amaral Filho e Slomski (2010), desde a década de 1960, o tema de controle de custos é tratado pela legislação brasileira, reportando-se à Lei Federal o 4.320/64. Porém, apesar da previsão legal, a cultura de controle de custos e, até mesmo, a Lei ainda não tinham sua aplicação de forma efetiva no setor público brasileiro, de acordo com o que apontam Alonso (1999), Mauss e Souza (2008) e Padrones et al. (2017).

Conforme Silva, Morgan e Costa (2004), a adoção de uma contabilidade de custos que promova evidenciação é complexa. Essa complexidade é sentida pelo nível de implantação reduzido. Para Machado e Holanda (2010), o panorama é de mudança quando "nos últimos anos o assunto deixou de ser [...] uma intenção (vontade), saindo da esfera da discussão das ideias para ser um processo estruturado com responsáveis, concepção teórica definida, cronograma e recursos".

O cenário de mudança é efetivado por meio da Resolução CFC nำ 1.129/2008, que aprovou a NBC T 16.2, a qual trata do Patrimônio e dos Sistemas Contábeis. A referida norma veio estabelecer que sistema contábil é a estrutura de informações sobre identificação, mensuração, avaliação, registro, controle e evidenciação dos atos e 
Percepções de especialistas sobre o processo de mudança na contabilidade de custos no setor público do Brasil

Renato Pereira Monteiro, Joaquim Carlos da Costa Pinho

dos fatos da gestão do patrimônio público (Conselho Federal de Contabilidade, 2011). A mesma norma reconheceu que essa estruturação tem por objetivo orientar e suprir o processo de decisão dentro das organizações, a prestação de contas e a instrumentalização do chamado controle social.

Pelo disposto no novo conjunto legal, a contabilidade do setor público passou a se organizar por meio de subsistemas que permitem controlar, gerir e evidenciar diferentes informações convergentes quanto ao objeto final que é o patrimônio público (CFC, 2011). A norma inseriu, junto aos demais subsistemas de informação (orçamentário, patrimonial e compensação), o subsistema de informação de custo - SICSP -, que tem como função registrar, processar e evidenciar os custos dos bens e serviços produzidos e ofertados à sociedade pela entidade, o qual foi posteriormente regulado pela Resolução no 1.366/2011, que aprovou a NBC T 16.11, que trata especificamente do SICSP e entrou em vigor no dia $1^{\circ}$ de janeiro de 2012. Com efeito, por meio dessa resolução, a contabilidade pública passou a ter um regramento a ser seguido para evidenciação e controle de seus custos.

O SICSP ainda tem sua importância ampliada em razão de sua formatação com os demais subsistemas, os quais devem ser integrados entre si e a outros subsistemas de informações, de modo a subsidiar a administração pública no que diz respeito ao desempenho da unidade contábil no cumprimento da sua missão, avaliação dos resultados obtidos na execução das ações do setor público com relação a aspectos como economicidade, eficiência, eficácia e efetividade; avaliação das metas estabelecidas pelo planejamento; avaliação dos riscos e das contingências e conhecimento da composição e movimentação patrimonial (CFC, 2011). Os avanços na contabilidade pública, segundo Machado e Holanda (2010), como adoção do regime de competência e implantação do sistema de custos, permitirão o incremento das possibilidades de melhorias na eficiência do gasto governamental do Brasil, mas alertam que o maior desafio está em "fazer com que as informações geradas sejam úteis e apropriadas ao processo decisório" (Machado, \& Holanda, 2010). 
Percepções de especialistas sobre o processo de mudança na contabilidade de custos no setor público do Brasil

Renato Pereira Monteiro, Joaquim Carlos da Costa Pinho

\subsection{Norma de Custos NBC T 16.11 - aspectos fundamentais}

Conforme a nova redação da norma, alterada em 2013, tem-se como fundamento estabelecer conceituação, objeto, objetivos e regras básicas para mensuração e evidenciação dos custos no setor público. O SICSP tem como objetivo criar uma nova realidade no setor público e permitir que ele organize suas informações de forma a permitir: (a) mensurar, registrar e evidenciar os custos de produtos, serviços, programas, projetos, atividades, ações, órgãos e outros objetos de custos da entidade; (b) apoiar a avaliação de resultados e desempenhos e permitir comparação entre os custos da entidade com os custos de outras entidades públicas, estimulando assim melhoria do desempenho dessas entidades; (c) apoiar a tomada de decisão em processos, tais como comprar ou alugar, produzir internamente ou terceirizar determinado bem ou serviço; (d) apoiar as funções de planejamento e orçamento e fornecer informações que permitam projeções mais aderentes à realidade com base em custos incorridos e projetados; (e) apoiar programas de controle de custos e de melhoria da qualidade do gasto (CFC, 2011).

Pode-se inferir que a norma não se distancia dos preceitos da fase da despesa pública e da forma como ela deve ser classificada, nos termos da Lei $n^{\circ} 4.320$, quando estabelece que a evidenciação dos objetivos dos custos também pode ser efetuada pela ótica institucional, funcional e programática (CFC, 2011). A norma pontua algumas recomendações para permitir o atingimento de seus objetivos. Prescreve que deve ter tratamento conceitual adequado, abordagem tecnológica apropriada, em que se propicie atuar com as múltiplas dimensões (temporais, numéricas e organizacionais, etc.) e se permita análise de séries históricas de custos sob a ótica das atividades-fim ou administrativas do setor público e, ainda, que se faça uso de ferramentas que permitam acesso rápido aos dados, tudo isso conjugado a tecnologias de banco de dados de forma a facilitar a criação de relatórios e a análise dos dados (CFC, 2011).

A norma ainda destaca o valor que a informação gerencial deve ter para as instituições do setor. Sua relevância para o interesse público pode ser entendida pelo seu impacto sobre a gestão pública, tanto do ponto de vista legal quanto de sua 
Percepções de especialistas sobre o processo de mudança na contabilidade de custos no setor público do Brasil

Renato Pereira Monteiro, Joaquim Carlos da Costa Pinho

utilidade. Para Lovato, Oliveira, Guimarães e Catapan (2016), o novo sistema do setor permite capturar os dados de outros sistemas a fim de realizar uma integração entre os processos de orçamento e planejamento, permitindo-lhe exercer maior controle sobre as despesas e sobre a mensuração e evidenciação dos custos das entidades públicas. No mesmo sentido, Lovato et al. (2016) afirmam que "é importante alinhar a implantação do SICSP com as necessidades e objetivos organizacionais, de forma sistemática e gradual [...]", e, para isso, deve-se compreender e ter em consideração a estrutura física da entidade.

A norma a ser utilizada quanto ao método de custeio deixa uma margem para escolha. Os principais métodos de custeio apontados pela norma são: direto; variável; por absorção; por atividade; pleno (CFC, 2011). A escolha do método deve estar apoiada na disponibilidade de informações e no volume de recursos necessários para a obtenção das informações ou dados (CFC, 2011). As entidades podem adotar mais de uma metodologia de custeamento, dependendo das características dos objetos de custeio (CFC, 2011). Porém ela não define com clareza qual dos métodos de custeio deve ser utilizado entre as opções existentes.

Diante deste cenário, constata-se que todo o processo de implantação já deveria ter ocorrido de forma mais efetiva depois da criação da norma, mas ele enfrenta dificuldades que retardam sua implantação, conforme apontam estudos anteriores como de Drehmer et al. (2016), Lovato et al. (2016) e Padrones et al. (2017).

\subsection{Dificuldades e barreiras para a implantação da contabilidade de custos no setor público}

Um fator considerado na literatura como barreira para implantação de novas normas contábeis é a cultura da organização e sua resistência a mudanças. Conforme Lapsley e Pallot (2000), a adoção de inovações de contabilidade de gestão implica mudança de comportamento e reflete a flexibilidade da entidade e de seus membros para aplicar ferramentas administrativas inovadoras. Semelhante ao apresentado por Fuertes (2008), é necessário considerar as barreiras derivadas dos diferentes 
Percepções de especialistas sobre o processo de mudança na contabilidade de custos no setor público do Brasil

Renato Pereira Monteiro, Joaquim Carlos da Costa Pinho

ambientes nacionais ao aplicar um novo modelo de contabilidade. Os valores subjacentes à cultura social, política e administrativa determinam uma série de características especiais nas práticas contábeis governamentais que fazem a convergência de fato.

Não distante desse entendimento, Arnaboldi e Lapsley (2009) destacam que o conflito político pode ser uma barreira para a implantação da contabilidade de exercício e, por conseguinte, também a de custos. Ao discorrer sobre o caso da Indonésia, VanPeursem e Eggleton (2015) relatam que as reformas sofrem influências por tradições históricas e pelo excesso de controle centralizado, o que as torna ainda mais complexas. No mesmo sentido, Benito et al. (2007) destacam que a mudança para padrões internacionais de contabilidade é um progresso longo, ao qual existe muita resistência.

A falta de detalhamento das normas internacionais é um dos pontos que pode gerar dificuldades em sua aplicação e interpretação como destacado por Catalano, Carlo e Erbacci (2015). Segundo esses autores, as IPSASs - International Public Sector Accounting Standards Board não fornecem quaisquer orientações detalhadas que possam ajudar quanto à superação dos problemas de registro e avaliação típicos do setor público.

Conforme Ball (2012), alguns obstáculos e dificuldades para a implantação das IPSAS podem ser também considerados para a contabilidade de custos, como políticas, restrições constitucionais e legais, e os recursos, que incluem pessoas e sistemas. $O$ mesmo autor considera que países em desenvolvimento terão mais dificuldades, pois esse obstáculo é real, uma vez que esses países carecem de mais recursos financeiros, extremamente necessários para um sistema de contabilidade por competência.

Semelhante a Ball (2012), Rossi et al. (2016), fazem referência a uma série de fatores que podem ser interpretados como dificuldades para a implantação de novas normas de contabilidade, inclusive, mas não restritamente, em relação à contabilidade de custos: (a) investimentos em tecnologia da informação e comunicações; (b) 
Percepções de especialistas sobre o processo de mudança na contabilidade de custos no setor público do Brasil

Renato Pereira Monteiro, Joaquim Carlos da Costa Pinho

treinamento de funcionários públicos; (c) custos relacionados à identificação e avaliação dos ativos na contabilidade por competência; (d) custos com consultorias.

Sobre os fatores constrangedores do processo de implantação, Machado e Holanda (2010) concluíram que "a mudança não se fará por decreto. Será preciso aperfeiçoamento contínuo do arcabouço conceitual e, sobretudo, mudanças de comportamentos".

Conclusões importantes sobre a busca por implementar o sistema de custos no governo federal do Brasil e suas dificuldades foram apontadas no estudo de Dantas e Crozatti (2014), por exemplo, que consideram precárias a atuação e a estrutura das setoriais contábeis com base nos resultados do estudo, que apresentam indicativos de que a alta administração não está envolvida no processo e projeto de implantação do sistema. Existe ainda a necessidade de maior apoio dos órgãos centrais para com os órgãos setoriais, a fim de subsidiar a implantação do sistema de forma adequada. Concluíram ainda que a informação de custos de forma geral não é utilizada, apesar de sua importância ser reconhecida.

\subsection{Teoria institucional}

A literatura atual aponta que as implantações das práticas de contabilidade de gestão no setor público têm como origem o processo de inovação da NPM, mas sua implantação ou não implantação pode ser explicada pelo uso da teoria institucional. Conforme Cunha, Dos Santos e Beuren (2015), "o processo de mudança das práticas e sistemas contábeis encontra, na análise institucional, subsídios valiosos à sua interpretação".

Conforme Guerreiro, Frezatti, Lopes e Pereira (2005) asseveram, o "processo de institucionalização de um novo sistema de contabilidade gerencial não é trivial e não pode ser enfocado apenas na dimensão técnica". Essa implantação depende de diversos fatores e deve haver comprometimento e compreensão dos indivíduos com sua importância, bem como depende da capacidade das pessoas envolvidas. Ela decorre de um processo de mudança de hábitos e rotinas dentro da organização, por 
Percepções de especialistas sobre o processo de mudança na contabilidade de custos no setor público do Brasil

Renato Pereira Monteiro, Joaquim Carlos da Costa Pinho

meio da reprodução de hábitos, ação e de pensamento, de acordo com Burns e Scapens (2000).

No setor público, essa tarefa ainda é mais complexa quando se trata do SIC, pois não depende exclusivamente da alteração na legislação. Sua disseminação tem como pressuposto a difusão de uma cultura gerencial no setor estatal que sobreponha os padrões de burocracia existentes, o que pode ser atingido por meio da reprodução de rotinas, pensamentos e ações com esse enfoque (Cunha et al., 2015). À luz da teoria institucional, Guerreiro et al., 2005), listam um conjunto de fatores que devem estar integrados para o avanço das modificações na contabilidade de gestão das organizações, conforme Quadro 1.

\begin{tabular}{|c|c|}
\hline & Fator \\
\hline 1 & Orientação normativa no sentido de implementação do novo modelo. \\
\hline 2 & O novo modelo deve ser efetivamente compreendido pelas pessoas. \\
\hline 3 & $\begin{array}{c}\text { Devem existir elementos facilitadores para o processo de repetição do modelo a fim de } \\
\text { proporcionar certa estabilidade e gerar hábitos. }\end{array}$ \\
\hline 4 & $\begin{array}{c}\text { As pessoas precisam perceber claramente as consequências da implantação do } \\
\text { modelo no plano pessoal. }\end{array}$ \\
\hline 5 & $\begin{array}{c}\text { As pessoas devem perceber claramente as consequências da implantação do modelo } \\
\text { no âmbito do grupo. }\end{array}$ \\
\hline 6 & $\begin{array}{c}\text { A implementação do novo modelo precisa conferir legitimidade para as pessoas, } \\
\text { interna e externamente. }\end{array}$ \\
\hline 7 & $\begin{array}{c}\text { O novo modelo necessita estar sintonizado com crenças e valores aceitos e } \\
\text { compartilhados pelos membros da organização. }\end{array}$ \\
\hline 8 & $\begin{array}{l}\text { O novo modelo precisa representar efetivamente como os indivíduos serão avaliados } \\
\text { não só institucionalmente, mas também subjetivamente pelos seus pares. }\end{array}$ \\
\hline
\end{tabular}

\section{Quadro 1. Fatores necessários à implantação de inovações contábeis}

Fonte: Guerreiro et al., 2015 (adaptado).

É possível perceber que as práticas de contabilidade de gestão acabam por ser adotadas conforme as expectativas dos indivíduos e em adequação às normas sociais de comportamento aceitáveis nas empresas, que acabam por se converter em rotinas aceitas pela organização. Essa adoção na prática não está muito vinculada aos melhores conceitos e técnicas impostos pela teoria (Guerreiro et al., 2005). 
Percepções de especialistas sobre o processo de mudança na contabilidade de custos no setor público do Brasil

Renato Pereira Monteiro, Joaquim Carlos da Costa Pinho

\subsection{Estudos empíricos anteriores sobre contabilidade de custos no setor público}

O estudo de Lovato et al. (2016) refere-se à implantação do SICSP em municípios do Estado do Paraná. A amostra era composta pelos contadores responsáveis das prefeituras num total de 399. O objetivo principal era investigar a percepção dos responsáveis pela implantação e utilização do SICSP. Obtiveram um total de 136 respostas. Os resultados mostraram que, em $40 \%$ dos municípios, a implantação do SICSP está num percentual $0 \%$. Para $50 \%$ dos respondentes, a implantação está entre 1\% e 75\% e apenas para 10\% a implantação está em fase de conclusão. Foi possível estabelecer uma relação entre o SICSP com os instrumentos de gestão e de ações gerenciais. Isso revelou, na opinião dos autores, a importância da implantação e sua utilidade. Entre as conclusões, está a existência de uma relação positiva obtida entre o percentual de implantação do SICSP e a quantidade de pessoas envolvidas com o projeto.

O estudo de Rosa, Da Silva e Soares (2015) teve como objetivo pesquisar a percepção de contadores de prefeituras municipais do estado de Santa Catarina sobre a implantação de um sistema de custos para a administração pública. A amostra era composta pelas 295 prefeituras, das quais responderam 97. Os autores concluíram que, para 97 respondentes, a implementação dos sistemas de custos é importante. As informações geradas são úteis ou muito úteis e "reconhecem sua utilidade, tanto no planejamento orçamentário quanto nas demais fases de atuação da administração pública, como na tomada de decisões e busca pela transparência [...]" (Rossi et al., 2016). O estudo corrobora o indicativo de baixo grau de implantação, uma vez que em apenas um município o sistema já estava implantado, e outros 14 indicaram estar em implantação.

A pesquisa de Carneiro, Mittmann, De Souza e Rodrigues (2013) foi realizada no município de Vilhena/RO e tinha por objetivo constatar os fatores que determinam as dificuldades para a implantação de um sistema de custos no município e analisar a percepção dos gestores e servidores sobre o conhecimento de sistema de custos. Foram realizadas 17 entrevistas com servidores, com formação na área de Ciências 
Percepções de especialistas sobre o processo de mudança na contabilidade de custos no setor público do Brasil

Renato Pereira Monteiro, Joaquim Carlos da Costa Pinho

Contábeis, da prefeitura e de autarquias municipais, partindo do reconhecimento de que Vilhena não tinha o SICSP implantado. Os autores concluíram que alguns aspectos podem dificultar a implantação do sistema de custos no município: (1) falta de conhecimento do gestor quanto ao uso da ferramenta; (2) falta de interesse desse tipo de controle; (3) falta de servidores especializados e capacitados (principalmente na área de tecnologia da informação); (4) falta de estudos na área; (5) falta de planejamento das atividades.

\section{METODOLOGIA}

Este estudo, quanto aos seus objetivos, é exploratório. Segundo Raupp e Beuren (2003), a pesquisa exploratória é aquela em que se tem pouco conhecimento sobre a temática e "busca-se conhecer com maior profundidade o assunto, de modo a torná-lo mais claro ou construir questões importantes para a condução da pesquisa". Essa classificação leva em consideração a necessidade de proporcionar um conjunto maior de informações sobre a implantação do SICSP, em especial sobre os potenciais fatores limitadores e dificuldades, algo que, nos estudos anteriores, não fica totalmente evidenciado e pode, no futuro, ser testado empiricamente.

Neste estudo, corroborando Lovato et al. (2016) e Rosa et al. (2015), optou-se por uma abordagem qualitativa como forma de permitir apropriar-se de questões ainda totalmente explicadas, relativas ao processo de implantação da contabilidade de custos no Brasil, semelhante ao aplicado por Carneiro et al. (2013), que também optou por uma abordagem qualitativa por meio de entrevistas.

Inicialmente, elaboraram-se as questões com o objetivo de realizar entrevistas pessoais com os autores. Todavia, após os primeiros contatos na tentativa de agendar a realização dessas entrevistas, percebeu-se o interesse dos autores em respondê-las por escrito, de acordo com o tempo e a disponibilidade de cada um. Neste sentido, o instrumento de coleta de dados que, antes, era um roteiro para entrevista semiestruturado, foi adaptado para um conjunto limitado de 10 questões. 
Percepções de especialistas sobre o processo de mudança na contabilidade de custos no setor público do Brasil

Renato Pereira Monteiro, Joaquim Carlos da Costa Pinho

Essa possibilidade de adaptação na forma de coletar os dados é uma das características da pesquisa qualitativa, em que as questões de investigação são sempre especificadas antes; podem surgir durante o estudo e de modo progressivo e atingir conclusões relevantes para a pesquisa (Sandelowski, 2008).

O critério de escolha dos especialistas foi identificar os autores de livros sobre contabilidade pública ou de custos no setor público com experiência na área contábil que tivessem publicado, no mínimo, um livro. A definição desse critério resultou em um total de onze obras de autores diferentes.

Após os contatos preliminares, nove especialistas se comprometeram a responder o questionário e assim efetivamente procederam. Um dos especialistas informou que não tinha interesse em responder, e outro deles não respondeu a nenhuma das tentativas de contato, via e-mail ou rede social.

Para preservar as opiniões apresentadas, não serão divulgados nominalmente os especialistas, somente o título de cada uma das obras dos onze selecionados para responder ao questionário. A ordem apresentada na análise das respostas de E1 até E9 não tem, propositalmente, relação com o ordenamento dos autores no quadro, a fim de preservar suas identidades.

\begin{tabular}{|c|c|c|}
\hline & Título do livro & Situação \\
\hline 01 & A nova contabilidade aplicada ao setor público & Respondeu \\
\hline 02 & Gestão de custos aplicada ao setor público & Respondeu \\
\hline 03 & Manual de controle patrimonial nas entidades públicas & Respondeu \\
\hline 04 & $\begin{array}{c}\text { Contabilidade pública: integrando união, estados e municípios (SIAFI e } \\
\text { SIAFEM) }\end{array}$ & Respondeu \\
\hline 05 & $\begin{array}{c}\text { Auditoria, contabilidade e controle interno no setor público: integração } \\
\text { das áreas do ciclo de gestão }\end{array}$ & Respondeu \\
\hline 06 & Entendendo as mudanças na contabilidade aplicada ao setor público & Respondeu \\
\hline 07 & Contabilidade pública na gestão municipal & Respondeu \\
\hline 08 & $\begin{array}{c}\text { Contabilidade pública: de acordo com as novas normas brasileiras de } \\
\text { contabilidade aplicadas ao setor público e à lei de responsabilidade } \\
\text { fiscal }\end{array}$ & Respondeu \\
\hline 09 & Contabilidade aplicada ao setor público: abordagem simples e objetiva & Respondeu \\
\hline
\end{tabular}

\section{Quadro 02. Livros publicados pelos especialistas}

Fonte: Dados da pesquisa (2016) 
Percepções de especialistas sobre o processo de mudança na contabilidade de custos no setor público do Brasil

Renato Pereira Monteiro, Joaquim Carlos da Costa Pinho

Os questionários foram enviados aos especialistas em Word® nos meses de agosto e setembro de 2014, solicitando-se que, no prazo de 30 dias, retornassem com as respostas.

O último questionário foi recebido no mês de fevereiro de 2015, após uma série de contatos. As repostas foram também recebidas em documento Word®. Os dados foram tratados da seguinte forma: (1) editados em planilha Excel®, sendo uma linha para cada resposta e uma coluna para cada especialista; (2) a planilha foi passada aos autores deste estudo, que grifaram os principais pontos de cada resposta para posterior discussão entre os autores dos pontos grifados de cada um; (3) quanto ao principal ponto, deveriam ser adotados os elementos que respondiam melhor à questão e não fugiam à pergunta em análise, e os trechos textuais mais relacionados com o enunciado. A análise dos dados partiu do seguinte protocolo: (1) tentar identificar pontos comuns, ou seja, elementos com os quais os autores concordavam ou que discordavam significativamente; (2) analisar e redigir a análise em cada questão de forma isolada, em alguns casos sendo necessário separar a opinião de cada especialista; (3) relacionar o conteúdo textual ou não com a categoria a que se referia a questão, como exposto no Quadro 3.

\begin{tabular}{|c|c|}
\hline Questão & Estágio ou fase de implantação \\
\hline 1 & Importância \\
\hline 2 & Pontos negativos e positivos na norma \\
\hline 3 & Necessidade de alteração \\
\hline 4 & Dificuldades \\
\hline 5 & Alternativas às dificuldades \\
\hline 6 & Práticas e experiências de sucesso \\
\hline 7 & Oportunidade de melhoria com base nas práticas do setor privado \\
\hline 8 & Potenciais contributos \\
\hline 10 & O futuro do SICSP \\
\hline
\end{tabular}

\section{Quadro 3. Relação da questão com a categoria ou o fator de análise}

Fonte: O autor (2016) 
Percepções de especialistas sobre o processo de mudança na contabilidade de custos no setor público do Brasil

Renato Pereira Monteiro, Joaquim Carlos da Costa Pinho

\section{DESCRIÇÃO E ANÁLISE DOS RESULTADOS}

Nesta seção, constam os principais achados do estudo com base nas respostas obtidas dos entrevistados, buscando-se estabelecer uma relação entre as respostas e a teoria.

\subsection{Descrição dos resultados}

\section{Questão 1 - 0 estágio atual da contabilidade de custos no setor público}

Existe praticamente um consenso entre os especialistas de que a implantação da contabilidade de custos está num estágio preliminar, introdutório. Na percepção dos respondentes, vai levar algum tempo para sua implantação efetiva em razão das barreiras existentes. Expressões como embrionária, fase de concepção, cenário de dúvidas, incipiente, estágio inicial e início do processo surgiram nas respostas. Existe o entendimento de que o estágio atual é o início de um processo que se tornou real com a chegada da NBC $T$ 16.11. Os respondentes (E5), (E6) e (E7) fizeram alusão específica ao surgimento da NBT C 16.11 como ponto fundamental neste processo. Os respondentes (E1) e (E9) lembraram que a Lei $n^{\circ} 4.320$, de 1964, já havia definido essa obrigatoriedade.

Para os respondentes (E6) e (E9), é preciso ainda resolver a questão da contabilidade patrimonial no setor público. Eles entendem que isso é uma barreira à contabilidade de custos.

O respondente (E2) destacou que, nos municípios, ela não está sendo aplicada, e raros são os casos em que existem tentativas neste sentido, apontando que, nos municípios, esse processo será muito complexo, pois existirá uma dependência para a implantação de uma cobrança pelos órgãos de controle. Para (E8) e (E9), existe ainda uma carência de pessoal, e (E8) aponta para a rotatividade de servidores e, ainda, para a falta de formação adequada nas universidades. 
Percepções de especialistas sobre o processo de mudança na contabilidade de custos no setor público do Brasil

Renato Pereira Monteiro, Joaquim Carlos da Costa Pinho

\section{Questão 2 - Importância da contabilidade de custos no setor público}

A Questão 2 buscava a opinião dos especialistas sobre a importância da contabilidade de custos. Nesse sentido, existe uma concordância de que ela é importante no contexto atual da gestão pública no Brasil. Expressões como "muito importante", "fundamental", "possibilita melhoria" e "vejo contribuições" surgiram nas respostas. A importância destacada pelos respondentes consistiu em diferentes fatores dos quais ganham destaque: mostrar a realidade (E1), comparações de resultados, serviços, setores, entidades (E1 e E3), melhorar a gestão (E2, E6, E8 e E9), melhorar a utilização dos recursos (E2, E7 e E9), efetividade, eficiência e eficácia (E7), planejamento (E2), tomada de decisão (E2, E3, E4 e E6), controle social (E3, E4 e E9), função alocativa (orçamento) (E6) e transparência pública (E7 e E9). Destoa do grupo a resposta de (E5): "Está servindo para atender recomendação do TCU de que deve existir" e este ainda critica a falta de efetividade de aplicação, "mas a falta de efetividade está em que o gestor não tem esta medida em sua avaliação pelos órgãos de controle". O especialista (E5) visualiza que o contexto atual atende somente a uma recomendação do órgão de controle (TCU) e que este deveria não só fiscalizar a existência ou não, mas a profundidade ou a cobrança de resultados de gestão neste sentido, o qual interpretamos como otimização dos recursos ou atendimento de indicadores de desempenho. A questão da importância está relacionada mais à existência ou não no resultado que o setor público é capaz de produzir.

\section{Questão 3 - Pontos negativos e positivos da NBC T 16.11}

A Questão 3 tinha o objetivo de identificar pontos negativos e positivos em relação à NBC $T$ 16.11. A análise desse conjunto de respostas foi feita tal como na questão sobre aspectos positivos e negativos. Em relação aos pontos positivos, podem ser assim agregados: boa redação, aspecto da inovação da norma; oferece o arcabouço conceitual e metodológico para a implantação dos sistemas de custos nas organizações públicas; estabelece o papel dos contabilistas no processo; traz o tema a debate; evidencia informações dos objetos de custo em notas explicativas; existe 
Percepções de especialistas sobre o processo de mudança na contabilidade de custos no setor público do Brasil Renato Pereira Monteiro, Joaquim Carlos da Costa Pinho

integração do sistema de custos com os demais sistemas organizacionais, principalmente planejamento e orçamento.

Quanto aos aspectos negativos, foram destacados: apenas conceitual; pode gerar confusão na aplicação (por ser apenas conceitual); não atende à necessidade de aplicação prática (por ser apenas conceitual); pouca norma efetivamente (seria necessário um detalhamento efetivo do sistema de informação de custos); falta definição ou, pelo menos, indicação de qual método de custeio deve ser adotado.

Um ponto de discordância entre os respondentes é o fato de ser apenas conceitual. Para (E1) e (E6), trata-se de um fator positivo. Os respondentes (E3), (E4) e (E8) consideram que o viés somente conceitual é um fator negativo, pois não atende à necessidade da implantação prática. Dois respondentes não identificam nenhum aspecto negativo na norma (E2 e E9).

\section{Questão 4 - Necessidade de alterações na norma}

Não existe um fator de consenso entre os respondentes. Quatro deles afirmaram que não deve sofrer alterações; três apontaram possíveis sugestões de alterações ou documentos complementares: criar um manual prático mostrando modelos e forma de aplicação do sistema de custos; reformular a responsabilidade do profissional contábil, uma vez que a implantação do sistema de custos está mais voltada para o gestor da entidade; padronizar de forma que haja a mesma contabilização em nível nacional ao indicar um método de custeio; excluir do texto toda a parte Demonstração do Resultado Econômico.

Outros respondentes destacaram que não deve haver alteração, uma vez que seu grande objetivo é de fortalecer o arcabouço conceitual e, na interpretação destes, a norma atende este objetivo, enfatizado por (E2), (E4), (E6) e (E9). Destaca-se a resposta de (E9), que não identifica necessidade de alterações, argumentando que o CFC, por meio do grupo assessor da área pública, do qual faz parte, estuda o assunto desde meados de 2008, tendo publicado a primeira versão da norma em 2011 e, em 2012 e 2013, foram publicados ajustes que aperfeiçoaram o texto. O atual estágio é de 
Percepções de especialistas sobre o processo de mudança na contabilidade de custos no setor público do Brasil

Renato Pereira Monteiro, Joaquim Carlos da Costa Pinho

disseminação do conteúdo e estímulo à implantação dos sistemas de custos. "Em termos de norma, acho que estamos bem, o que precisamos é avançar com o conhecimento junto aos profissionais e gestores da área pública do País".

Já para (E5), a norma não responde à necessidade do setor, pois trata da sistemática e não da efetividade, e (E1) não se posicionou em relação às alterações, pois não havia aplicado a norma, mas indicou a necessidade de "o CFC, junto com a STN, elaborarem um manual prático, mostrando modelos e forma de aplicação do sistema de custos".

\section{Questão 5 - Dificuldades para a implantação}

A Questão 5 perguntava aos entrevistados sobre qual ente da Federação terá mais dificuldades em implantar com sucesso a contabilidade de custos e por quê. $O$ entendimento de que a grande dificuldade estará nos municípios, em especial os de pequeno porte, é defendido por (E7), (E1), (E9) e (E4), em razão de fatores como falta de recursos e pessoal qualificado, rotatividade, falta de sistema informatizado, desconhecimento dos gestores e segregação de funções inadequadas. Na União, a dificuldade é percebida em razão da grandiosidade da estrutura, de acordo com (E3). Para (E2), a dificuldade é a mesma. Se, por um lado, a União tem mais recursos, a tarefa é mais complexa, ao contrário dos municípios. Segundo (E5), "A dificuldade está no fato de que os entes federativos estão procurando desenvolver o sistema patrimonial. Só depois pensarão em custos". Segundo (E6), "todos terão dificuldades, pois pouquíssimos ou quase nenhum fazem uma boa contabilidade patrimonial. ". A contabilidade patrimonial não é o único obstáculo apresentado por (E6) que ainda considera que entidades com menos recursos financeiros terão problemas para desenvolver seus sistemas (se forem próprios) e aqueles que farão esta contratação do setor privados, enfrentarão dificuldades pela carência de profissionais capacitados para manipular a informação disponível. 
Percepções de especialistas sobre o processo de mudança na contabilidade de custos no setor público do Brasil

Renato Pereira Monteiro, Joaquim Carlos da Costa Pinho

\section{Questão 6 - Alternativa às dificuldades}

A Questão 6 tratava das alternativas para superar as dificuldades referentes à implantação do SICSP. Os entrevistados apontaram uma série de medidas que podem ser adotadas pelo setor, tais como: profissionalizar os chefes do Executivo, treinar as pessoas e buscar apoio direto da União; implementar eficientes sistemas de controle e auditoria internos; elaborar e aplicar um projeto de capacitação nacional e desenvolver sistemas informatizados que atendam às demandas geradas pela informação de custo; bons sistemas informatizados de contabilidade e profissionais bem remunerados, bem como aprovar uma lei de âmbito nacional, definindo piso salarial para contador da área pública; alinhar CFC e Tribunais de Contas para exigirem controle num mesmo padrão; criar norma contábil ou lei definindo aplicação de multa aos gestores que não implementarem condições de controle de custos; promover mudança em termos de vontade política e, na sequência, o envolvimento dos profissionais de contabilidade no processo, sendo estes, de alguma forma, responsáveis pelo processo.

\section{Questão 7 - Práticas e experiência de sucesso}

Conforme (E1), a região Sul do país está mais adiantada em relação à aplicação de todas as normas de contabilidade, porém essa afirmação necessita de maior validade empírica, pois confronta com os resultados de estudos como o de Lovato et al. (2016). Ao menos no que se refere à norma de custos, o processo ainda está em fase muito inicial. O respondente (E2) afirmou que tem apoiado a implantação com sucesso nos municípios, em especial nas áreas de educação e saúde, e identifica como modelos de sucesso o estado do Rio Grande do Sul e o município do Rio de Janeiro. Outros respondentes colocaram casos específicos de municípios que teriam a implantação em estágio avançado e com boas práticas, como o caso da Prefeitura de Caxias do Sul/RS e a prefeitura de Recife/PE, que teriam o sistema em operação desde 2011. Porém, na ampla afirmação dos autores, seriam casos isolados. 
Percepções de especialistas sobre o processo de mudança na contabilidade de custos no setor público do Brasil

Renato Pereira Monteiro, Joaquim Carlos da Costa Pinho

\section{Questão 8 - Aprendizagem com o setor privado}

Os especialistas acreditam que o setor público tem no setor privado um conjunto de práticas que pode auxiliar no sentido de melhoria em seu processo de controle de custos, conforme (E1), (E2) e (E3). Eles destacam que o setor privado está mais avançado neste sentido. Para (E4), o aprendizado deve ser o que fazer com a informação, pois, uma vez implantada, resta saber o que fazer com ela. Daí o principal desafio é fazer "Gestão de Custos", conforme (E4). Para (E6), deve existir uma gestão mais voltada e preocupada com resultados como forma de atender a sociedade e the prestar contas. No entendimento de (E8), além do resultado no profissionalismo e controle, considera que "é fundamental buscar o melhor resultado nos programas de governo, pois isso gera sobra de recursos para outras ações, bem como melhor aplicação dos recursos públicos e, por consequência, mais capacidade de investimentos do ente público".

\section{Questão 9 - Contributos da implantação}

A Questão 9 visava a identificar quais são as principais contribuições que a contabilidade de custos pode trazer para o país. Nesse ponto, foram destacados pelos respondentes elementos como: evidenciar os custos de bens e serviços produzidos e oferecidos à sociedade; permitir uma comparação do que é recolhido a título de imposto e o que retorna para a sociedade: economicidade, eficiência, eficácia, produtividade, transparência e decisões mais seguras por parte dos gestores para criar um controle financeiro efetivo.

No que diz respeito a municípios, surgiu avaliação de custo-benefício, como e quando gastar, apoio ao controle interno e suporte indispensável para as decisões estratégicas, informações já alimentadas pelos sistemas orçamentário e patrimonial, plano de contas único, centralizar as informações dentro da contabilidade, economia de recursos, melhor investimento dos recursos, tomada de decisões, governança pública. 
Percepções de especialistas sobre o processo de mudança na contabilidade de custos no setor público do Brasil

Renato Pereira Monteiro, Joaquim Carlos da Costa Pinho

\section{Questão 10 - Futuro da Contabilidade de Custos}

Neste ponto, se destacam algumas respostas que apontam a tendência de que serão muitas as dificuldades, que o processo será demorado, mas que a mudança é uma exigência da sociedade. Para (E1), "é um trabalho de 'formiguinha', mas acredito que a sociedade como um todo vem mudando e pedindo mais transparência nos gastos da administração pública. Não teremos de imediato a aplicação de todas as normas, mas pelo que vejo muitos estão trabalhando para que isso aconteça. Dessa forma é uma questão de tempo para quebrarmos essa barreira". Segundo (E2), "vamos tentar implantar os sistemas. É uma exigência da sociedade em termos de transparência e divulgação das ações públicas". Já (E3) se posiciona afirmando que "é um processo lento, que não será integralmente aplicado de imediato. Será ampliado com o tempo, com a disseminação de conhecimento, exigências de órgãos fiscalizadores e quebras de paradigmas. Em termos de entes como União e Estado, a meu ver, o destino tenderá a gerar resultado em médio prazo. Em termos de municípios e suas administrações indiretas, minha visão será de longo prazo. Para onde ir é aplicação, um destino de todos. Os processos são vários e envolvem sistemas, pessoas, tempo e fluxos diversos".

Os especialistas sugerem que esse processo avançará gradativamente em velocidades distintas em cada ente da federação, muito por força de certa coerção social em busca de transparência e responsabilização dos gestores.

\subsection{Discussão dos resultados}

É sensível que, para os especialistas, o estágio de implantação é inicial e preliminar e que deverá decorrer muito tempo para haver avanços mais significativos. Esses apontamentos se alinham com os resultados de estudos empíricos recentes realizados, como Rosa et al. (2015), Lovato et al. (2016) e Araújo, Carneiro e Santana (2016).

Os especialistas destacaram que a NBC T 16.11 foi importante no processo de implantação, corroborando com Vicente et al. (2012). Todavia, chamam a atenção para 
o sistema de custos, que já estava previsto na Lei ํㅡ 4.320, de 1964, como enfatizado por Slomski et al. (2010). Parece que o panorama de mudança previsto por Machado e Holanda (2010) está ocorrendo, mas em uma velocidade muito reduzida, inclusive em âmbito de órgãos federais, como constataram Padrones et al. (2017).

Os contributos do SICSP apontados pelos especialistas ratificam o destacado na literatura como forma de melhorar o conjunto de informações para a tomada de decisão. Esses contributos se alinham com os objetivos do SICSP previstos na normativa do CFC, como é o caso da função alocativa da informação de servir ao controle social e às funções de planejamento. Estes se alinham com o posicionamento de autores como Benito et al. (2007), Arnaboldi e Lapsley (2009) e, em parte, com os achados de Rosa et al. (2015): planejamento, transparência pública e tomada de decisão.

Sobre os pontos de destaque da norma, percebeu-se que existe o entendimento de que ela traz avanços ao quadro anterior. Consolida um arcabouço conceitual necessário para introduzir a contabilidade de custos e gerar o debate. Preenche uma lacuna existente; traz os instrumentos necessários para a implantação da contabilidade de custos, mas percebe-se que, como afirmado por Guerreiro et al. (2005), as introduções de inovações na contabilidade de gestão não dependem exclusivamente do processo de normalização, pois são mais complexas. Ao contrário, o processo já estaria em maior nível de evolução, mas, como já referido, os estudos empíricos recentes apontam no sentido oposto.

As principais críticas centram-se em relação ao seu papel (conceitual) e à não identificação de qual método deve ser adotado, argumentos que corroboram o apontado por Catalano et al. (2015).

Entre as possíveis alterações que a norma deveria sofrer, não existe um consenso. Nosso posicionamento é alinhado com os respondentes (E2), (E4), (E6) e (E9), no sentido de que a norma cumpre seu papel. Porém só a norma não fará a contabilidade de custos ser implantada, e isso tem respaldo na teoria institucional e, por exemplo, no quadro descrito por Guerreiro et al. (2005) dos fatores que devem estar 
Percepções de especialistas sobre o processo de mudança na contabilidade de custos no setor público do Brasil

Renato Pereira Monteiro, Joaquim Carlos da Costa Pinho

integrados para alterações dessa ordem nas organizações (ver Quadro 1) e também nas dificuldades que se elencam, a começar pela mudança de mentalidade no que diz respeito a tomar decisões com base em informações de custos. Essa carência decorre em muito da falta de cultura no controle de custos, já descrita por Alonso (1999), Maus e Souza (2008) e Padrones et al. (2017).

Sobre as dificuldades apontadas, existe um entendimento, de boa parte dos especialistas, de que os municípios menores têm mais condicionantes ao processo de implantação, pois algumas barreiras para a implantação são mais frequentes nestes por suas características como falta de recursos (materiais, financeiros e humanos), perfil dos gestores e acúmulo indevido de funções. Esse aspecto corrobora o entendimento mais geral na literatura internacional, como em Smith (2004), Van Dooren (2005) e Gomes, Carvallho e Fernandes (2009), uma vez que os municípios maiores têm mais recursos (financeiros e humanos). Por outro lado, Pereira e Romão (2016) identificaram mesmos percentuais de implantação em municípios de pequena e grande dimensão (no caso de Portugal) e assumem que "contrariamente ao que poderia ser expectável, a variável dimensão não é condição essencial para a implementação do SIC". Nosso entendimento é de que isso precisa ser explorado em relação à realidade brasileira, pois a abordagem (em termos de volume de receitas) feita por Lovato et al. (2016) também não identificou essa relação que está presente para parte dos entrevistados.

Um dos pontos que pode ser referido como um fator que motivará a adoção será o maior rigor institucional dos órgãos de controle com ações mais punitivas aos gestores como forma de combater as restrições à implantação.

Os principais contributos da contabilidade de custos para o setor repetem, em parte, os fatores de importância já referidos, alinhados em muito com os objetivos descritos pela norma de custos. Nosso posicionamento é no sentido de que serão dois os contributos centrais, um destinado ao usuário interno e outro ao usuário externo: melhora na decisão por parte dos gestores e transparência. Ambos têm a mesma origem, num conjunto de informações de custos disponível, formatável, útil e seguro, que não só atenda à obrigação legal, mas tenha utilidade para a tomada de decisão. 
Percepções de especialistas sobre o processo de mudança na contabilidade de custos no setor público do Brasil

Renato Pereira Monteiro, Joaquim Carlos da Costa Pinho

Sobre a conjuntura para o futuro do processo de implantação, o cenário credível na opinião dos especialistas é de continuidade nas dificuldades, mas que existe uma pressão social em busca da transparência. Assim a continuidade do processo é um caminho sem volta. Isso será possível com disseminação do conhecimento e exigências específicas e punições para além da quebra de paradigma, como identificou o respondente (E3). Esse entendimento está alinhado com a teoria institucional e também com o caminho percorrido por outros países neste sentido, como o caso de Portugal, descrito por Pereira e Romão (2016), em que, mesmo passados mais de dez anos da normativa (sobre a contabilidade de custos em Portugal), os níveis de implantação ainda não são satisfatórios (pouco superiores a 50\%).

\section{CONCLUSÃO}

O estudo teve por objetivo responder: (1) Como os especialistas entendem o processo de implantação da NBC T 16.11?; (2) Qual é a utilidade e importância desse sistema? (3) Quais são as principais dificuldades encontradas? (4) Qual é a perspectiva de futuro?

Pode-se identificar que existe o entendimento de que a implantação do SICSP está em fase muito inicial, o que corrobora os estudos empíricos realizados recentemente, e deverá existir um esforço coletivo em termos de formação, mudança de mentalidade e estruturação do setor para permitir a consolidação da implantação. Neste cenário, a norma cumpre um papel de promover o debate e ser um marco regulatório-conceitual para sua implantação, semelhante ao realizado em outros países. Porém, somente o processo de normatização não confere efetividade ao processo de implantação, justificando as referências sobre a teoria institucional nas alterações da contabilidade de gestão em organizações.

A importância do SICSP é relevada no entendimento dos especialistas sobre sua possível utilização, o que se assemelha às características com as quais a NPM pondera para o setor público e com os próprios objetivos descritos na literatura e referidos no 
Percepções de especialistas sobre o processo de mudança na contabilidade de custos no setor público do Brasil

Renato Pereira Monteiro, Joaquim Carlos da Costa Pinho

contexto da NBC T 16.11, tais como: melhoria na tomada de decisão, prestação de contas, planejamento do orçamento e controle. Os especialistas apontaram como de extrema importância sua implantação para esclarecer como os recursos públicos são aplicados, para melhorar a gestão das entidades e racionalizar o uso dos recursos.

Percebe-se ainda que existem barreiras para essa implantação, que surgem por motivos como falta de vontade política, falta de treinamento dos servidores públicos, a questão da remuneração e rotatividade dos profissionais do setor, o sistema de informática utilizado, despreparo dos municípios e suas dificuldades, falta de cultura de controle de custos, falta de cobrança de exigência dos órgãos de controle e carência de uma contabilidade patrimonial adequada. Alguns especialistas apontaram que os municípios menores têm maior dificuldade em relação à carência de alguns recursos (em especial financeiro).

Essas barreiras definem o longo caminho a ser percorrido para a implantação e superação desses fatores restritivos. Porém, a sociedade exige mais transparência do setor público, e caberá aos gestores encontrar soluções para atender a nova demanda social. Infere-se ainda que uma forma de avançar com os níveis de implantação será a maior cobrança por parte das entidades fiscalizadoras (tribunais de contas), inclusive com punições (multas) pelo descumprimento da norma.

O estudo tem como limitação retratar a realidade do Brasil e ser um estudo ainda exploratório, com limitações pertinentes à abordagem qualitativa adotada em razão das dificuldades existentes em realizar a análise das respostas, tendo em vista que se trata de questões abertas. Tais fatores afetam a análise das respostas e as conclusões realizadas, e isso incide na análise, por exemplo, do nível de concordância em relação a importância ou das dificuldades destacadas. Esta interpretação das respostas parte do entendimento dos autores do estudo, que acabam por inferências com base em seus próprios pressupostos e entendimentos do tema, incidindo em certa subjetividade.

É possível e necessário realizar novos estudos que busquem confrontar as respostas aqui obtidas com a realidade de estudos empíricos atuais realizados sobre a implantação da norma no Brasil, por exemplo, por meio de uma seleção no Congresso 
Percepções de especialistas sobre o processo de mudança na contabilidade de custos no setor público do Brasil

Renato Pereira Monteiro, Joaquim Carlos da Costa Pinho

Brasileiro de Custos, a fim de verificar se as dificuldades aqui elencadas são relatadas por outros autores.

Percebe-se ainda que as características dos municípios (porte, capacidade financeira, entre outros) e sua relação com o nível de implantação ainda não foram diagnosticados na realidade brasileira.

\section{REFERÊNCIAS}

Alonso, M. (1999). Custos no serviço público. Revista Do Serviço Público, 50(1), 37-63.

Araújo, C. A. de., Carneiro, A. de F., \& Santana, A. F. B. (2016). Sistemas de Custos Públicos: Entendimento e Implantação nos Municípios de Rondônia. Revista de Contabilidade do Mestrado em Ciências Contábeis, UERJ, 20(2).

Arnalboldi, M., \& Lapsley, I. (2009). On the Implementation of Accrual Accounting: A Study of Conflict and Ambiguity. European Accounting Review, 18(4), 809-836.

Ball, I. (2012). New development: Transparency in the public sector. Public Money \& Management, 32(1), 35-40.

Benito, B., Brusca, I., \& Montesinos, V. (2007). The harmonization of government financial information systems: the role of the IPSASs. International Review of Administrative Sciences, 73(2), 293-317.

Burns, J., \& Scapens, R. W. (2000). Conceptualizing management accounting change: an institutional framework. Management Accounting Research, 11(1), 3-25, doi: https://doi.org/http://dx.doi.org/10.1006/mare.1999.0119

Catalano, G., Carlo, F. Di, \& Erbacci, A. (2015). Accrual accounting in Italian universities: a technical perspective. International Journal of Public Sector Management, 28(6), 494-508.

Carneiro, A. F., Mittmann, R. A. A. C., De Souza, J. A., \& Rodrigues, S. (2013). Sistema de custos municipal: Desafios, obstáculos e percepção no caso do município de Vilhena, Rondônia. Revista Ambiente Contábil, 5(2), 53.

Cunha, P. R., Dos Santos, V., \& Beuren, I. M. (2015). Artigos de periódicos internacionais que relacionam teoria institucional com contabilidade gerencial. Perspectivas Contemporâneas, 10(2), 1-23. 
Percepções de especialistas sobre o processo de mudança na contabilidade de custos no setor

público do Brasil

Renato Pereira Monteiro, Joaquim Carlos da Costa Pinho

Drehmer, A. F., Raupp, F. M., \& Tezza, R. (2016, Novembro). Implantação do sistema de informação de custos em prefeituras catarinenses: o que dizem os contadores sobre as dificuldades e contribuições? Anais do Congresso Brasileiro de Custos, Porto de Galinhas, PE, 23.

Dantas, J. M. M., \& Crozatti, J. (2014). Implantação do sistema de custos no governo federal: percepção da maturidade das setoriais de custos. Cadernos de Finanças Públicas, (14).

Dasí, R. M., Montesinos, V., \& Murgui S. (2013). Comparative Analysis of Governmental Accounting Diversity in the European Union. Journal of Comparative Policy Analysis: Research and Practice, 15(3), 255-273, doi: <https://doi.org/10.1080/13876988.2013.798117

Fuertes, I. (2008). Towards Harmonization or Standardization in Governmental Accounting? The International Public Sector Accounting Standards Board Experience. Journal of Comparative Policy Analysis: Research and Practice, 10(4), 327-345.

Gomes, P., Carvallho, J., \& Fernandes, M. (2009). Determinantes da adoção e desenvolvimento do sistema de contabilidade de custos nos Municípios Portugueses. Contabilidade e Gestão, 8, p. 11-40.

Guerreiro, R., Frezatti, F., Lopes, A. B., \& Pereira, C. A. (2005). O entendimento da contabilidade gerencial sob a ótica da teoria institucional. Organizações \& Sociedade, 12(35), 91-106.

Lapsley, I., \& Pallot, J. (2000). Accounting, management and organizational change: A comparative study of local government. Management Accounting Research, 11(2), 213-229.

Lei Complementar no 101, de 04 de maio de 2000. Estabelece normas de finanças públicas voltadas para a responsabilidade na gestão fiscal e dá outras providências. In: Diário Oficial [da] República Federativa do Brasil. Brasília, 05 de maio de 2000. Brasil: LRF.

Lei no 10.180, de 06 de fevereiro de 2001. Organiza e disciplina os Sistemas de Planejamento e de Orçamento Federal, de Administração Financeira Federal, de Contabilidade Federal e de Controle Interno do Poder Executivo Federal e dá outras providências. Diário Oficial [da] República Federativa do Brasil. Brasília, 07 de fevereiro de 2001.

Lei no 4.320, de 17 de março de 1964. Estatui normas gerais de direito financeiro para elaboração e controle dos orçamentos e balanços da União, Estados, Municípios e 
Percepções de especialistas sobre o processo de mudança na contabilidade de custos no setor público do Brasil

Renato Pereira Monteiro, Joaquim Carlos da Costa Pinho

do Distrito Federal. Diário Oficial [da] República Federativa do Brasil. Brasília, 23 de março de 1964.

Lovato, E. L., Oliveira, A. G. de, Guimarães, I. A., \& Catapan, A. (2016, Janeiro - Abril). Sistemas de Informações de Custos no Setor Público no Brasil: Uma Análise da Percepção dos Usuários Sob o Enfoque da Gestão Governamentaly. Revista Globalización, Competitividad y Gobernabilidad, 10(1), 16-41.

Machado, N., \& Holanda, V. B. de. (2010). Diretrizes e modelo conceitual de custos para o setor público a partir da experiência do governo federal. Revista de Administração Pública, 44(4), 791-820.

Mauss, C. V., \& Souza, M. A. de. (2008). Gestão de custos aplicado ao setor público: modelo para mensuração e análise da eficiência e eficácia governamental. São Paulo: Atlas.

Monteiro, R. P., Pereira, C. A., \& Souza, M. A. de. (2015). A necessidade da informação gerencial nas IES públicas da Europa: breve abordagem pela realidade de Portugal. Revista Catarinense Da Ciência Contábil, 14(42), 9-24.

Monteiro, R. P., Pereira, C. A., \& Pereira, N. S. P. (2014). O impacto das reformas da administração pública brasileira na regulação contabilística do setor. Revista UNEMAT de Contabilidade, 3(6).

Oulasvirta, L. (2014). The reluctance of a developed country to choose International Public Sector Accounting Standards of the IFAC. A critical case study. Critical Perspectives on Accounting, 25(3), 272-285.

Padrones, G. S. A., Santos, W. J. L. dos., \& Colares, A. C. V. (2017). Implantação do Sistema de Informação de Custos no setor público: A experiência do Governo Federal Brasileiro. Science of Human Action, 2(1), 68-98.

Pangnani \& Oliveira, J. (2009, Novembro). Administração e gestão de custos em saneamento básico: um estudo de empresa pública municipal. Anais do Congresso Brasileiro de Custos, Fortaleza, CE, 16.

Pereira, A. L., \& Romão, A. L. (2016). A implementação do sistema de contabilidade de custos nos municípios portugueses. Revista Contemporânea de Contabilidade, 12(27), 165-186.

Pina, V., Torres, L., \& Yetano, A. (2009). Accrual Accounting in EU Local Governments: One Method, Several Approaches. European Accounting Review, 18(4), 765-807. 
Percepções de especialistas sobre o processo de mudança na contabilidade de custos no setor

público do Brasil

Renato Pereira Monteiro, Joaquim Carlos da Costa Pinho

Raupp, F. M., \& Beuren, I. M. (2003). Metodologia da pesquisa aplicável às ciências sociais. Como elaborar trabalhos monográficos em contabilidade: teoria e prática, 3 , p. 76-97.

Resolução no 1.129, de 21 de novembro de 2008. Aprova a NBC T 16.2-patrimônio e sistemas contábeis." Diário Oficial da União, Poder Executivo, Brasília, DF 25 (2008). Contabilidade, Conselho Federal - CFC.

Resolução CFC n. 1.366, de 25 de novembro de 2011. Aprova a NBC T 16.11-sistema de informação de custos do setor público." Diário Oficial da União, Poder Executivo, Brasília, DF 2 (2011). CFC - Conselho Federal de Contabilidade.

Rosa, F. S., Da Silva, L. C., \& Soares, S. V. (2015). Aspectos de convergência da contabilidade no setor público: um enfoque sobre a implantação do sistema de custos segundo a percepção de contadores municipais do estado de Santa Catarina/International Public Sector Accounting Standards: the perception munic. Contabilidad y Negocios, 10(19), 27.

Rossi, F. M., Cohen, S., Caperchione, E., \& Brusca, I. (2016). Harmonizing public sector accounting in Europe: thinking out of the box. Public Money \& Management, 36(3), 189-196.

Sandelowski, M. J. (2008). Justifying qualitative research. Research in nursing \& health, 31(3), 193-195.

Silva, C. A. T., Morgan, B. F., \& Costa, P. de S. (2004, Julho). Apuração do custo de ensino por aluno: aplicação a uma instituição federal de ensino superior. Anais do Encontro Nacional dos Programas de Pós-Graduação em Administração, Curitiba, PR, p. 15.

Slomski, V., CAMARGO, G. B. de, Amaral Filho, A. C. C. do., \& Slomski, V. G. (2010). A demonstração do resultado econômico e sistemas de custeamento como instrumentos de evidenciação do cumprimento do princípio constitucional da eficiência, produção de governança e accountability no setor público: uma aplicação na Procuradoria Geral do Município de São Paulo. Revista de Administração Pública, 44(4), 933-57.

Smith, K. A. (2004). Voluntarily reporting performance measures to the public a test of accounting reports from US Cities. International Public Management Journal, 7(1), 19.

Van Dooren, W. (2005). What makes organisations measure? Hypotheses on the causes and conditions for performance measurement. Financial accountability \& management, 21(3), 363-383. 
Van Peursem, K., \& Eggleton, I. R. C. (2015). Indonesian public sector accounting reforms: dialogic aspirations a step too farnull. Accounting, Auditing \& Accountability Journal, 28(5), 706-738.

Vicente, E. F. R., Morais, L., Platt, N. O. A. (2012). A reforma na contabilidade pública brasileira e o processo de convergência: Implicações e perspectivas. RIC-Revista de Informação Contábil, 6(2), 01-20.

Data de Submissão: 13/09/2016

Data de Aceite: 28/06/2017 